\title{
Perimeter as relaxed Minkowski content in metric measure spaces
}

\author{
Luigi Ambrosio $^{*} \quad$ Simone Di Marino ${ }^{\dagger} \quad$ Nicola Gigli ${ }^{\ddagger}$
}

July 4, 2018

\begin{abstract}
In this note we prove that on general metric measure spaces the perimeter is equal to the relaxation of the Minkowski content w.r.t. convergence in measure.
\end{abstract}

\section{Introduction}

In a metric measure space $(X, \mathrm{~d}, \mathfrak{m})$, the upper and lower Minkowski contents are respectively defined by

$$
\mathcal{M}_{-}(A):=\liminf _{r \downarrow 0} \frac{\mathfrak{m}\left(A^{r}\right)-\mathfrak{m}(A)}{r}, \quad \mathcal{M}_{+}(A):=\limsup _{r \downarrow 0} \frac{\mathfrak{m}\left(A^{r}\right)-\mathfrak{m}(A)}{r}
$$

for Borel sets $A$ with finite $\mathfrak{m}$-measure. The Minkowski contents, even in their non-infinitesimal versions, appear in many areas, as in the theory of concentration of measures and isoperimetric inequalities (see for instance [Led], CM15a, CM15b] and the references therein) and the theory of random closed sets AmCaVi].

For sufficiently nice metric measure structures, the relations between Minkowski content and perimeter are well-known, see for instance $\$ 2.13$ in AFP and $\S 14.2$ in [BuZa. Aim of this note is the investigation of more precise relations between the Minkowski content and the perimeter, as defined in the theory of $B V$ functions in metric measure spaces. In particular we prove in Lemma 2.1 and Theorem 3.6 that the lower semicontinuous envelope w.r.t. $L^{1}(X, \mathfrak{m})$ convergence of the Minkowski contents $\mathcal{M}_{ \pm}(A)$ is equal to the perimeter $\operatorname{Per}(A)$. As a byproduct, we can prove that in metric measure spaces with finite $\mathfrak{m}$-measure the Cheeger constant

$$
\gamma:=\inf \left\{\frac{\mathcal{M}_{+}(A)}{\mathfrak{m}(A)}: 0<\mathfrak{m}(A) \leq \frac{\mathfrak{m}(X)}{2}\right\}
$$

can be equivalently defined replacing $\mathcal{M}_{+}(A)$ with $\mathcal{M}_{-}(A)$, or with $\operatorname{Per}(A)$.

Another consequence of our result is that whenever one wants to establish, on a given space, an isoperimetric inequality of the form

$$
\mathfrak{m}(A) \leq f(\operatorname{Per}(A))
$$

\footnotetext{
${ }^{*}$ Scuola Normale Superiore, Pisa. email: luigi.ambrosio@sns.it

${ }^{\dagger}$ Laboratoire de Math. d'Orsay, Univ. Paris-Sud, CNRS, Univ. Paris-Saclay. email: sthew87@gmail.com

${ }^{\ddagger}$ SISSA, Trieste. email: ngigli@sissa.it
} 
for some continuous non-decreasing function $f: \mathbb{R}^{+} \rightarrow \mathbb{R}^{+}$, it is sufficient to prove the easier

$$
\mathfrak{m}(A) \leq f\left(\mathcal{M}_{+}(A)\right) \text {. }
$$

We remark that not only it is easily seen that $\mathcal{M}_{+}(A) \geq \operatorname{Per}(A)$ (see also the proof of Theorem [3.6), but also that the Minkowski content is a quantity that by nature is sometimes handled better than the perimeter in estimates involving the geometry of the space. An example in this direction is the recent paper [CM15a] by Cavalletti-Mondino, which motivated our study (see also the work in progress [CM15c], which contains results similar to ours, under curvature assumptions).

Another goal of the paper is a closer investigation of the coarea formula and of the "generic" properties of superlevel sets of Lipschitz functions. In Euclidean and other nice spaces, the combination of the Fleming-Rishel formula (involving the perimeter of superlevel sets) with the coarea formula for Lipschitz maps (involving the Hausdorff measure of level sets) provides many useful informations, even on the level sets, as illustrated in Remark 4.1. Under a suitable regularity assumption (4.2) on the metric measure structure, fulfilled in all spaces $R C D(K, \infty)$ of AGS11b, we provide in Proposition 4.2 a metric counterpart of this, involving the Minkowski contents. Finally, we are able to make a more detailed analysis for level sets of distance functions and we conclude the paper pointing out a few open questions.

\section{Basic setting and preliminaries}

Throughout this paper $(X, \mathrm{~d})$ is a metric space and $\mathfrak{m}$ is a nonnegative and $\sigma$-additive measure on its Borel $\sigma$-algebra; we always assume that $\mathfrak{m}$ is finite on bounded Borel sets. In particular, $\mathfrak{m}$ is $\sigma$-finite.

In the metric space $(X, \mathrm{~d})$ we denote by $B_{r}(x)$ the open ball with center $x$ and radius $r$. We denote by $\operatorname{Lip}(f)$ the Lipschitz constant of a Lipschitz function $f: X \rightarrow \mathbb{R}$ and we will often use the distance function

$$
\mathrm{d}_{A}(x):=\inf _{y \in A} \mathrm{~d}(x, y)
$$

from a nonempty set $A$, whose Lipschitz constant is less than 1 . The slope $\operatorname{lip}(f)$ (also called local Lipschitz constant) of $f: X \rightarrow \mathbb{R}$ is defined by

$$
\operatorname{lip}(f)(x):=\limsup _{y \rightarrow x} \frac{|f(y)-f(x)|}{\mathrm{d}(y, x)},
$$

with the convention $\operatorname{lip}(f)(x)=0$ if $x$ is an isolated point.

We denote by $\chi_{A}: X \rightarrow\{0,1\}$ the characteristic function of a set $\mathrm{A}$ and we say that $A_{h} \rightarrow A$ in $\mathfrak{m}$-measure if $\int_{X}\left|\chi_{A_{h}}-\chi_{A}\right| d \mathfrak{m} \rightarrow 0$ (equivalently, $\mathfrak{m}\left(A_{h} \Delta A\right) \rightarrow 0$ ). For any nonempty set $A \subset X$ and any $r>0$ we define the open $r$-enlargement $A^{r}$ of $A$ by

$$
A^{r}:=\left\{x \in X: \mathrm{d}_{A}(x)<r\right\} .
$$

Notice that $A^{r}=(\bar{A})^{r}$, and that the triangle inequality gives the semigroup inclusion

$$
\left(A^{s}\right)^{t} \subset A^{s+t} \quad s, t>0 .
$$

For any Borel set $A \subset X$ with $\mathfrak{m}(A)<\infty$ we define the upper and lower Minkowski contents by

$$
\mathcal{M}_{-}(A):=\liminf _{r \downarrow 0} \frac{\mathfrak{m}\left(A^{r}\right)-\mathfrak{m}(A)}{r}, \quad \mathcal{M}_{+}(A):=\limsup _{r \downarrow 0} \frac{\mathfrak{m}\left(A^{r}\right)-\mathfrak{m}(A)}{r} .
$$


Obviously one has $0 \leq \mathcal{M}_{-}(A) \leq \mathcal{M}_{+}(A) \leq \infty$. In addition, $\mathcal{M}_{-}(A)=\infty$ if $\mathfrak{m}(\bar{A} \backslash A)>0$, hence a natural domain for the Minkowski content is the class of essentially closed sets. Notice also that $\mathcal{M}_{-}(A)<\infty$ implies $\mathfrak{m}\left(A^{r} \backslash A\right) \rightarrow 0$ as $r \downarrow 0$.

We define the relaxed Minkowski contents $\mathcal{M}_{-}^{*}(A), \mathcal{M}_{+}^{*}(A)$ of $A$ by

$$
\begin{aligned}
& \mathcal{M}_{-}^{*}(A):=\inf \left\{\liminf _{h \rightarrow \infty} \mathcal{M}_{-}\left(A_{h}\right): A_{h} \rightarrow A \text { in m-measure }\right\}, \\
& \mathcal{M}_{+}^{*}(A):=\inf \left\{\liminf _{h \rightarrow \infty} \mathcal{M}_{+}\left(A_{h}\right): A_{h} \rightarrow A \text { in m-measure }\right\} .
\end{aligned}
$$

It is obvious that $\mathcal{M}_{-}^{*}(A) \leq \mathcal{M}_{+}^{*}(A)$. The following elementary lemma shows that equality holds, so from now on we will also use the notation $\mathcal{M}^{*}(A)$ for their common value.

Lemma 2.1 For any Borel set $A \subset X$ one has

$$
\mathcal{M}_{-}(A) \geq \mathcal{M}_{+}^{*}(A)
$$

In particular $\mathcal{M}_{-}^{*}(A)=\mathcal{M}_{+}^{*}(A)$.

Proof. We can assume with no loss of generality $\mathcal{M}_{-}(A)<\infty$, hence $\mathfrak{m}\left(A^{s} \backslash A\right) \rightarrow 0$ as $s \downarrow 0$. We start from the semigroup inclusion (2.1) to get

$$
\frac{\mathfrak{m}\left(A^{s+t}\right)-\mathfrak{m}\left(A^{s}\right)}{t} \geq \frac{\mathfrak{m}\left(\left(A^{s}\right)^{t}\right)-\mathfrak{m}\left(A^{s}\right)}{t} .
$$

Now, at any differentiability point $s$ of the nondecreasing and left continuous map $f(r):=$ $\mathfrak{m}\left(A^{r}\right)$ we get $f^{\prime}(s) \geq \mathcal{M}_{+}\left(A^{s}\right)$ and an integration w.r.t. $s$ gives

$$
\mathfrak{m}\left(A^{r}\right)-\mathfrak{m}(A)=f(r)-f\left(0_{+}\right) \geq \int_{0}^{r} f^{\prime}(s) d s \geq \int_{0}^{r} \mathcal{M}_{+}\left(A^{s}\right) d s \quad \forall r>0 .
$$

Dividing both sides by $r$ and letting $r \downarrow 0$ an application of the mean value theorem gives the first claim.

In order to prove the equality of relaxed Minkowski contents, let $A_{h} \rightarrow A$ in m-measure and apply (2.2) to get

$$
\mathcal{M}_{+}^{*}(A) \leq \liminf _{h \rightarrow \infty} \mathcal{M}_{+}^{*}\left(A_{h}\right) \leq \liminf _{h \rightarrow \infty} \mathcal{M}_{-}\left(A_{h}\right) .
$$

The arbitrariness of $A_{h}$ yields the inequality $\mathcal{M}_{+}^{*}(A) \leq \mathcal{M}_{-}^{*}(A)$.

\section{$3 \quad$ Equality of relaxed contents and perimeter}

In this section we shall use the semigroup

$$
T_{t} f(x):=\sup _{B_{t}(x)} f, \quad T_{0} f=f,
$$

which extends the operator $A \mapsto A^{t}$ from characteristic functions to functions, i.e. $T_{t} \chi_{A}=\chi_{A^{t}}$ for all $t>0$. Actually the term "semigroup" is not totally correct here, since only the inequality $T_{s+t} f \geq T_{s}\left(T_{t} f\right)$ holds for the operator $T_{t}$ in (3.1); the inequality is an equality 
if $(X, \mathrm{~d})$ is a length space (see also AmDi] for the simple proof and an example of strict inequality). We will only need the inequality in the sequel.

Notice that $T_{t} f \geq f$ and that $T_{t} f$ is lower semicontinuous for all $t>0$. The definition of $\operatorname{lip}(f)$ gives also immediately

$$
\limsup _{t \downarrow 0} \frac{T_{t} f-f}{t} \leq \operatorname{lip}(f)
$$

Remark 3.1 The semigroup $T_{t}$ already had a role (up to a change of sign, resulting in the replacement of sup with inf) in the proof given in AmDi] of the equality between relaxed upper gradients and "measure" upper gradients in the context of the theory of $B V$ functions and sets of finite perimeter. The "inf" semigroup can also be formally viewed as the limit as $p \rightarrow \infty$ of the semigroups

$$
T_{t}^{p} f(x):=\inf _{y \in X} f(y)+\frac{1}{p t^{p-1}} \mathrm{~d}^{p}(y, x) .
$$

associated to the Hamilton-Jacobi equation $\partial_{t} g+|\nabla g|^{q} / q=0$, see [AGS11a, GRS].

It is also worthwhile to mention that the semigroup $T_{t}$ has already been used in connection with the (anisotropic) Minkowski content in [CLL, but only in an Euclidean setting: their case can be regarded as our construction in the metric space $\left(\mathbb{R}^{d}, \mathrm{~d}_{C}, \mathscr{L}^{d}\right)$, where $\mathrm{d}_{C}$ is the gauge function associated to a convex set $C$.

Lemma 3.2 For all $f: X \rightarrow[0, \infty)$ Lipschitz with $\mathfrak{m}\left(\{f>0\}^{h}\right)<\infty$ for some $h>0$, one has

$$
\int_{0}^{\infty} \mathcal{M}_{-}(\{f \geq t\}) d t \leq \liminf _{t \downarrow 0} \int_{X} \frac{T_{t} f-f}{t} d \mathfrak{m} \leq \int_{X} \operatorname{lip}(f) d \mathfrak{m} .
$$

Proof. We start from the elementary "coarea" pointwise identities $\int_{0}^{\infty} \chi_{\{f \geq t\}} d t=f$ and

$$
\int_{0}^{\infty} T_{h} \chi_{\{f \geq t\}} d t=\int_{0}^{\infty} \chi_{\{f \geq t\}^{h}} d t=T_{h} f
$$

for $f: X \rightarrow[0, \infty]$. If $f$ is Borel with $\int_{X} f d \mathfrak{m}<\infty$, subtracting the first identity from the second one and integrating w.r.t. $\mathfrak{m}$ gives

$$
\int_{0}^{\infty} \frac{\mathfrak{m}\left(\{f \geq t\}^{h}\right)-\mathfrak{m}(\{f \geq t\})}{h} d t=\int \frac{T_{h} f-f}{h} d \mathfrak{m} .
$$

Now, notice that one has the bound

$$
\frac{T_{h} f-f}{h} \leq \operatorname{Lip}(f) \chi_{\{f>0\}^{h}} .
$$

Hence, assuming in addition that $f$ is Lipschitz and that for some $h>0$ we have $\mathfrak{m}(\{f>$ $\left.0\}^{h}\right)<\infty$, Fatou's lemma, dominated convergence and (3.2) give (3.3).

The following definition of perimeter, first proposed in Mi and further investigated in AmDi (dropping the local compactness assumption on the metric structure) is by now well studied, see also the following remarks.

Definition 3.3 (Perimeter) Let $A \subset X$ be a Borel set with $\mathfrak{m}(A)<\infty$. We define

$$
\operatorname{Per}(A):=\inf \left\{\liminf _{h \rightarrow \infty} \int_{X} \operatorname{lip}\left(f_{h}\right) d \mathfrak{m}: f_{h} \in \operatorname{Lip}(X), \lim _{h \rightarrow \infty} \int_{X}\left|f_{h}-\chi_{A}\right| d \mathfrak{m}=0\right\} .
$$


If $\mathfrak{m}(X)$ is finite we can also consider the perimeter of $X \backslash A$; it is then easy to check that $\operatorname{Per}(A)=\operatorname{Per}(X \backslash A)$. In the following remarks we show that smaller classes than $\operatorname{Lip}(X)$ can be considered in the definition of $\operatorname{Per}(A)$, comparing also with the definitions in [Mi] and AmDi.

Remark 3.4 A simple truncation argument shows that we need only to consider sequences $f_{h}$ with $0 \leq f_{h} \leq 1$ in (3.4). Also, if $\bar{x} \in X$ and $\phi_{R}: X \rightarrow[0,1]$ are 1-Lipschitz functions with $\phi_{R} \equiv 1$ on $B_{R}(\bar{x})$ and $\phi_{R} \equiv 0$ on $X \backslash B_{2 R}(\bar{x})$, from the inequality

$$
\int_{X} \operatorname{lip}\left(f_{h} \phi_{R}\right) d \mathfrak{m} \leq \int_{X} \operatorname{lip}\left(f_{h}\right) d \mathfrak{m}+\int_{X \backslash B_{R}(\bar{x})} f_{h} d \mathfrak{m}
$$

and a diagonal argument (i.e. choosing $R=R(h)$ ) we obtain that only sequences of Lipschitz functions $f_{h}: X \rightarrow[0,1]$ with $\left\{f_{h}>0\right\}$ bounded need to be considered in the definition of $\operatorname{Per}(A)$.

Remark 3.5 Definition 3.3 appeared first in [Mi] in complete and doubling metric measure spaces, requiring approximation by locally Lipschitz functions and $L_{\mathrm{loc}}^{1}$ convergence. Then, at the general level of complete and separable metric spaces (for instance the most appropriate setting for the theory of $C D(K, \infty)$ spaces), the definition has been revisited in AmDi], requiring $L^{1}$ convergence of $f_{h}$ to $\chi_{A}$, as in (3.4), but requiring $f_{h}$ to be locally Lipschitz (i.e. for any $x \in X$ there exists $r>0$ such that $\left.f\right|_{B_{r}(x)}$ is Lipschitz). As in [Mi], the motivation for this definition is that, when localized to open sets $U \subset X$ via

$$
\mu(U):=\inf \left\{\liminf _{h \rightarrow \infty} \int_{U} \operatorname{lip}\left(f_{h}\right) d \mathfrak{m}: f_{h} \in \operatorname{Lip}_{\mathrm{loc}}(U), \lim _{h \rightarrow \infty} \int_{U}\left|f_{h}-\chi_{A}\right| d \mathfrak{m}=0\right\}
$$

is continuous w.r.t. monotone nondecreasing sequences and thus it provides the restriction to open sets of a finite Borel measure, namely the perimeter measure.

In proper metric spaces (a class that includes complete and doubling metric spaces) it is not hard to prove that all these variants of Definition 3.3 lead to the same definition, for sets with finite $\mathfrak{m}$-measure. The equivalence persists also for complete and separable spaces, but the proof is not elementary, see Section 4 and, in particular, Theorem 4.5.3 in [Di]. However, the equivalence with AmDi] does not play any role in the paper and Definition 3.3 will be our working definition.

Theorem 3.6 For any Borel set $A \subset X$ with $\mathfrak{m}(A)<\infty$ one has $\mathcal{M}_{-}^{*}(A)=\operatorname{Per}(A)$.

Proof. Claim: $\mathcal{M}_{-}(A) \geq \operatorname{Per}(A)$. We can assume with no loss of generality $\mathcal{M}_{-}(A)<\infty$, hence $\mathfrak{m}\left(A^{r} \backslash A\right) \rightarrow 0$ as $r \downarrow 0$. Let $\tau \in(0,1)$ be fixed and let $f_{r}: X \rightarrow[0,1]$ be given by

$$
f_{r}(x)=1-1 \wedge \frac{\mathrm{d}_{A^{s}}(x)}{r^{\prime}} \quad r>0,
$$

where $s>0$ satisfies $s+r^{\prime}<r$. Since $A$ is contained in the open set $A^{s}$, where $f_{r}$ is identically equal to 1 , one has $\operatorname{lip}\left(f_{r}\right) \equiv 0$ on $A$; on the other hand, since the complement of $A^{r}$ is contained in the open set $\left\{d_{A^{s}}>r^{\prime}\right\}$, where $f_{r}$ is identically equal to $0, \operatorname{lip}\left(f_{r}\right) \equiv 0$ on the complement of $A^{r}$. Thus, the inequalities $\operatorname{lip}\left(f_{r}\right) \leq \operatorname{Lip}\left(f_{r}\right) \leq 1 / r^{\prime} \leq 1 /(\tau r)$ give

$$
\frac{\mathfrak{m}\left(A^{r}\right)-\mathfrak{m}(A)}{r} \geq \tau \int_{X} \operatorname{lip}\left(f_{r}\right) d \mathfrak{m} .
$$


We now estimate

$$
\int_{X}\left|f_{r}-\chi_{A}\right| d \mathfrak{m}=\int_{X \backslash A} f_{r} d \mathfrak{m} \leq \mathfrak{m}\left(A^{r} \backslash A\right) \rightarrow 0 .
$$

Then, choosing an infinitesimal sequence $\left(r_{h}\right)$ of radii on which the liminf is achieved, since $f_{r_{i}} \rightarrow \chi_{A}$ in $L^{1}(X, \mathfrak{m})$ the very definition of $\operatorname{Per}(A)$ gives $\mathcal{M}_{-}(A) \geq \tau \operatorname{Per}(A)$. Eventually we let $\tau \uparrow 1$.

Claim: $\mathcal{M}_{-}^{*}(A) \geq \operatorname{Per}(A)$. Let $A_{h} \rightarrow A$ in $\mathfrak{m}$-measure and use the lower semicontinuity of the perimeter and the above claim to get

$$
\operatorname{Per}(A) \leq \liminf _{h \rightarrow \infty} \operatorname{Per}\left(A_{h}\right) \leq \liminf _{h \rightarrow \infty} \mathcal{M}_{-}\left(A_{h}\right) .
$$

Since $A_{h}$ are arbitrary, this proves that $\operatorname{Per}(A) \leq \mathcal{M}_{-}^{*}(A)$.

Claim: $\operatorname{Per}(A) \geq \mathcal{M}_{-}^{*}(A)$. Thanks to Remark 3.4 we can find a family of Lipschitz functions $f_{h}: X \rightarrow[0,1]$ with $\left\{f_{h}>0\right\}$ bounded, $f_{h} \rightarrow \chi_{A}$ in $L^{1}(X, \mathfrak{m})$ and $\int_{X} \operatorname{lip}\left(f_{h}\right) d \mathfrak{m} \rightarrow \operatorname{Per}(A)$. For all $\epsilon \in(0,1 / 2)$ we can find, thanks to (3.3), $t_{h} \in(\epsilon, 1-\epsilon)$ with

$$
\mathcal{M}_{-}\left(\left\{f_{h} \geq t_{h}\right\}\right) \leq \frac{1}{1-2 \epsilon} \int_{X} \operatorname{lip}\left(f_{h}\right) d \mathfrak{m} .
$$

Since $\left\{f_{h} \geq t_{h}\right\} \rightarrow A$ in $\mathfrak{m}$-measure it follows that $(1-2 \epsilon) \mathcal{M}_{-}^{*}(A) \leq \operatorname{Per}(A)$ and letting $\epsilon \downarrow 0$ the inequality is achieved.

\section{Level sets of Lipschitz functions}

In this section we study the relation between perimeter and Minkowski content for generic superlevel sets of Lipschitz functions.

Definition 3.3 of perimeter is a particular case of the following one, for $f \in L^{1}(X, \mathfrak{m})$ :

$$
\operatorname{Var}(f):=\inf \left\{\liminf _{h \rightarrow \infty} \int_{X} \operatorname{lip}\left(f_{h}\right) d \mathfrak{m}: f_{h} \in \operatorname{Lip}(X), \lim _{h \rightarrow \infty} \int_{X}\left|f_{h}-f\right| d \mathfrak{m}=0\right\} .
$$

The two concepts are closely related. Indeed, by approximating any $L^{1}$ function with step functions and, conversely, characteristic functions of $\{f>t\}$ by $\chi_{\varepsilon} \circ f$, where $\chi_{\epsilon}$ is a smooth approximation of $\chi_{[t, \infty)}$, it can be easily proved (see [Mi] for details) that the classical coarea formula of Fleming-Rishel, namely

$$
\operatorname{Var}(f)=\int_{0}^{\infty} \operatorname{Per}(\{f \geq t\}) d t \quad f \in L^{1}(X, \mathfrak{m}), f \geq 0
$$

holds even in this abstract setting, without any finiteness assumption on either side of the equality. Under the regularity assumption

$$
\operatorname{Var}(f)=\int_{X} \operatorname{lip}(f) d \mathfrak{m} \quad \text { for all } f \in L^{1}(X, \mathfrak{m}) \text { Lipschitz }
$$

on the metric measure structure we can now prove that we can replace perimeter with the lower Minkowski content in (4.1), namely

$$
\operatorname{Var}(f)=\int_{0}^{\infty} \mathcal{M}_{-}(\{f \geq t\}) d t \quad f \in L^{1}(X, \mathfrak{m}), f \geq 0 .
$$


Remark 4.1 In Euclidean spaces $\mathbb{R}^{n}$, combining the Fleming-Rishel formula, the pointwise inequalities

$$
\operatorname{Per}(\{f \geq t\})=\mathscr{H}^{n-1}\left(\partial^{*}\{f \geq t\}\right) \leq \mathscr{H}^{n-1}(\partial\{f>t\}) \leq \mathscr{H}^{n-1}(\{f=t\})
$$

(where $\partial^{*}\{f \geq t\}$ is the essential boundary of $\{f \geq t\}$, a countably $\mathscr{H}^{n-1}$-rectifiable set whenever $\operatorname{Per}(\{f \geq t\})$ is finite) and the coarea formula for nonnegative Lipschitz functions

$$
\int_{\mathbb{R}^{n}}|\nabla f| d x=\int_{0}^{\infty} \mathscr{H}^{n-1}(\{f=t\}) d t
$$

one obtains

$$
\operatorname{Per}(\{f \geq t\})=\mathscr{H}^{n-1}(\partial\{f \geq t\})=\mathscr{H}^{n-1}(\{f=t\}) \quad \text { for } \mathscr{L}^{1} \text {-a.e. } t>0
$$

and that $\{f=t\}$ is countably $\mathscr{H}^{n-1}$-rectifiable for $\mathscr{L}^{1}$-a.e. $t>0$. However, the validity of (4.3) is a nontrivial information even in the Euclidean case, since rectifiability does not imply, in general, finiteness of the Minkowski content and agreement with the Hausdorff measures. See $\S 2.13$ of $[\mathrm{AFP}$ for an example of compact countable set having infinite Minkowski content. Finiteness of the Minkowski contents is ensured for instance by density lower bounds of the form

$$
\sigma\left(B_{r}(x)\right) \geq \frac{\mathfrak{m}\left(B_{r}(x)\right)}{r} \quad \forall x \in \partial A, r \in(0,1)
$$

for some finite measure $\sigma$, see Theorem 2.104 for the simple argument, which also proves that this property, in conjunction with rectifiability, provides agreement of the Minkowski contents with the Hausdorff measures.

Notice that, thanks to the truncation argument of Remark 3.4, an equivalent formulation of (4.2) is the lower semicontinuity of the functional $f \mapsto \int_{X} \operatorname{lip}(f) d \mathfrak{m}$, restricted to Lipschitz and integrable functions, w.r.t. $L^{1}(X, \mathfrak{m})$ convergence.

Proposition 4.2 Under assumption (4.2), one has

$$
\operatorname{Var}(f)=\int_{0}^{\infty} \mathcal{M}_{-}(\{f \geq t\}) d t
$$

for any Lipschitz function $f: X \rightarrow[0, \infty)$ with $\mathfrak{m}\left(\{f>0\}^{h}\right)<\infty$ for some $h>0$. As a consequence

$$
\mathcal{M}_{-}(\{f \geq t\})=\operatorname{Per}(\{f \geq t\}) \quad \text { for } \mathscr{L}^{1} \text {-a.e. } t>0 .
$$

In addition, if $f: X \rightarrow[0, M]$ is 1 -Lipschitz, $\mathfrak{m}\left(\{f>0\}^{h}\right)<\infty$ for some $h>0$ and $\operatorname{lip}(f)=1$ $\mathfrak{m}$-a.e. in $\{0<f<M\}$, one has

$$
\mathcal{M}_{-}(\{f \geq t\})=\mathcal{M}_{+}(\{f \geq t\}) \quad \text { for } \mathscr{L}^{1} \text {-a.e. } t \in(0, M) .
$$

Proof. Taking (4.1) and the inequality $\mathcal{M}_{-}(A) \geq \operatorname{Per}(A)$ into account, for the first part of the statement it is sufficient to prove the inequality $\geq$ in (4.4). Since by assumption $\operatorname{Var}(f) \geq \int_{X} \operatorname{lip}(f) d \mathfrak{m}$, the inequality follows at once from (3.3) of Lemma 3.2.

In order to prove the second statement we start from the inequality

$$
\frac{\mathfrak{m}\left(\{f \geq t\}^{h}\right)-\mathfrak{m}(\{f \geq t\})}{h} \leq \frac{\mathfrak{m}(\{f \geq t-h\})-\mathfrak{m}(\{f \geq t\})}{h}
$$


to get $\mathcal{M}_{+}(\{f \geq t\}) \leq-\mathfrak{m}(\{f \geq t\})^{\prime}$ for $\mathscr{L}^{1}$-a.e. $t>0$. By integration in $(0, M)$ we get

$$
\int_{0}^{\infty} \mathcal{M}_{+}(\{f \geq t\}) d t \leq \mathfrak{m}(\{0<f<M\}) \leq \int_{X} \operatorname{lip}(f) d \mathfrak{m}=\int_{0}^{\infty} \mathcal{M}_{-}(\{f \geq t\}) d t
$$

whence the result follows.

Remark 4.3 (The case of distance functions) Assume that $(X, \mathrm{~d})$ satisfies the following length assumption: for any $x, y \in X$ there exists an $\epsilon$-geodesic between $x$ and $y$, namely a finite collection of points $z_{0}, \ldots, z_{N}$ with $z_{0}=x, z_{N}=y, \max _{0 \leq i<N} \mathrm{~d}\left(z_{i}, z_{i+1}\right)<\varepsilon$ and

$$
\sum_{i=0}^{N-1} \mathrm{~d}\left(z_{i}, z_{i+1}\right)<\mathrm{d}(x, y)+\varepsilon .
$$

If $A \subset X$ is nonempty and closed, under this length assumption on $(X, \mathrm{~d})$ it is easy to check that $\operatorname{lip}\left(\mathrm{d}_{A}\right) \equiv 1$ in $X \backslash A$. Therefore, the second part of Proposition 4.2 is applicable to all functions $\left(M-\mathrm{d}_{A}\right)^{+}$for all $M>0$ and provides, still under the assumption (4.2), the property

$$
\mathcal{M}_{-}\left(\left\{\mathrm{d}_{A} \leq t\right\}\right)=\mathcal{M}_{+}\left(\left\{\mathrm{d}_{A} \leq t\right\}\right)=\operatorname{Per}\left(\left\{\mathrm{d}_{A} \leq t\right\}\right)
$$

for $\mathscr{L}^{1}$-a.e. $t>0$. If $\mathfrak{m}(X)<\infty$ we can apply the statement also to $\mathrm{d}_{A}$ to get

$$
\mathcal{M}_{-}\left(\left\{\mathrm{d}_{A} \geq t\right\}\right)=\mathcal{M}_{+}\left(\left\{\mathrm{d}_{A} \geq t\right\}\right)=\operatorname{Per}\left(\left\{\mathrm{d}_{A} \geq t\right\}\right)
$$

for $\mathscr{L}^{1}$-a.e. $t>0$. Since $\operatorname{Per}\left(\left\{\mathrm{d}_{A} \geq t\right\}\right)=\operatorname{Per}\left(\left\{\mathrm{d}_{A} \leq t\right\}\right)$ with at most countably many exceptions, the quantities in (4.5) and (4.6) coincide $\mathscr{L}^{1}$-a.e. in $(0, \infty)$.

If the length assumption above is enforced to

$\forall x, y \in X, \forall \varepsilon>0$ there exists $\gamma \in C([0,1] ; X)$ with $\gamma_{0}=x, \gamma_{1}=y, \operatorname{length}(\gamma)<\mathrm{d}(x, y)+\varepsilon$

(notice that the two length properties are equivalent in separable spaces), a simple continuity argument gives

$$
\left\{\mathrm{d}_{A}=t\right\}^{r} \backslash\left\{\mathrm{d}_{A}=t\right\}=\left\{0<\mathrm{d}_{\left\{\mathrm{d}_{A} \geq t\right\}}<r\right\} \cup\left\{0<\mathrm{d}_{\left\{\mathrm{d}_{A} \leq t\right\}}<r\right\} \quad \forall t \in(0, \infty), r>0 .
$$

Then, since $\mathfrak{m}\left(\left\{\mathrm{d}_{A}=t\right\}\right)=0$ with at most countably many exceptions, we obtain existence of the Minkowski content of the level set $\left\{\mathrm{d}_{A}=t\right\}$

$$
\lim _{r \downarrow 0} \frac{\mathfrak{m}\left(\left\{\mathrm{d}_{A}=t\right\}^{r}\right)}{2 r}=\operatorname{Per}\left(\left\{\mathrm{d}_{A} \geq t\right\}\right)=\operatorname{Per}\left(\left\{\mathrm{d}_{A} \leq t\right\}\right)
$$

for $\mathscr{L}^{1}$-a.e. $t>0$.

Concerning assumption (4.2), we point that it holds on doubling $\operatorname{RCD}(K, \infty)$ spaces (see AGS11b]) for the definition of the latter. Indeed, on one side we know by Rajala's paper [Ra] that on these spaces a 1-1 weak Poincaré inequality holds, so that thanks to Cheeger's results in Chee we have that the $p$-weak gradient is $\mathfrak{m}$-a.e. equal to the local Lipschitz constant of Lipschitz functions for $p>1$. On the other hand, in $\mathrm{GiHa}$ it has been proved that on $\operatorname{RCD}(K, \infty)$ spaces the $p$-weak gradients all coincide even for $p=1$ (this result is based on the Bakry-Emery estimate in the form established by Savaré in [Sa]), thus giving the claim. We illustrate in the next remark the known relations between $\operatorname{lip}(f)$ and other weak notions of gradient, and why doubling\&1-Poincaré alone are not sufficient to ensure the validity of (4.2). 
Remark 4.4 Recall that one of the key results of the seminal paper Chee is the lower semicontinuity of the functional $\int_{X} \operatorname{lip}(f)^{p} d \mathfrak{m}$ w.r.t. $L^{p}$ convergence on locally Lipschitz functions, for all $p \in(1, \infty)$, assuming the doubling property of the metric measure structure and the validity of a $p$-Poincaré inequality. This result can also be rephrased by saying that $\operatorname{lip}(f)$ coincides $\mathfrak{m}$-a.e. with the minimal $p$-weak upper gradient.

However, under the same structural assumptions, the result is not true in general when $p=1$. Indeed, revisiting an example by Carbone-Sbordone CaSb, in HKLL14, a metric measure space $(X, \mathrm{~d}, \mathfrak{m})$ is built as follows: $X=[0,1]$ is endowed with the Euclidean distance and with the weighted Lebesgue measure $\mathfrak{m}=\omega \mathscr{L}^{1}$, with $\omega \equiv 1$ on a "fat" Cantor set $K \subset(0,1)$ (i.e. a compact totally disconnected set with positive Lebesgue measure) and $\omega=1 / 2$, say, on $(0,1) \backslash K$. It is clear that $(X, \mathrm{~d}, \mathfrak{m})$, being comparable to the standard Euclidean structure, satisfies the doubling and 1-Poincaré assumptions. On the other hand, in [HKLL14] Lipschitz functions $f_{n}$ convergent to the identity function $f$ in $L^{1}(X, \mathfrak{m})$ are built in such a way that

$$
\limsup _{n \rightarrow \infty} \int_{X} \operatorname{lip}\left(f_{n}\right) d \mathfrak{m}<\int_{X} \operatorname{lip}(f) d \mathfrak{m},
$$

thus disproving the lower semicontinuity. This corresponds to the fact that two notions of minimal 1-weak upper gradients are possible in the $H^{1,1}$ (and $B V$ ) theory. The two notions do not coincide in general, and $\operatorname{lip}(f)$ coincides only with one of them (see [HKST, Theorem 12.5.1]). See also AmPiSp and [HKLL14 for a more detailed discussion.

\section{Some open problems}

In complete metric measure spaces with a doubling measure, under the assumption of the validity of a 1-Poincaré inequality, the first author proved in Am1, AM2 that the perimeter measure of Remark 3.5 coincides con $\theta \mathscr{S}^{h} L \partial^{*} E$, where $\partial^{*} E$ is the essential boundary of $E$ (i.e. the complement of the union of density and rarefaction points of $E$ ) and $\mathscr{S}^{h}$ is the measure built of out the gauge function $\zeta\left(\bar{B}_{r}(x)\right)=\mathfrak{m}\left(\bar{B}_{r}(x)\right) /(2 r)$ with Carathéodory's construction. The density $\theta$ is bounded from below by the structural constants involved in the doubling and Poincaré assumptions, but little more is known in general about it. Under additional regularity assumptions, it would be interesting to relate more closely the Minkowski content of $E$ with the measure $\mathscr{S}^{h}\left\llcorner\partial^{*} E\right.$, as in the Euclidean-Riemannian theory.

In the same vein, one can prove the coarea inequality (Proposition 5.1 in [AM2])

$$
\int_{0}^{\infty} \mathscr{S}^{h}(B \cap\{f=t\}) d t \leq \operatorname{Lip}(f) \mathfrak{m}(B) \quad B \text { Borel }
$$

reminiscent of (4.4): we propose here a self-contained proof of an improved version of this inequality, involving the asymptotic Lipschitz constant

$$
\operatorname{Lip}_{a}(f, x):=\lim _{r \downarrow 0} \operatorname{Lip}\left(f, B_{r}(x)\right)
$$

and, with an additional factor 2, the slope.

Proposition 5.1 Assume that $\mathfrak{m}$ is a doubling measure in the metric space $(X, \mathrm{~d})$. Let $f$ : $X \rightarrow[0, \infty)$ be a Lipschitz function with $\mathfrak{m}(\{f>0\})<\infty$. Then for every $B \subset X$ Borel one has

$$
\int_{0}^{\infty} \mathscr{S}^{h}(B \cap\{f=t\}) d t \leq \int_{B} \operatorname{Lip}_{a}(f, \cdot) d \mathfrak{m},
$$




$$
\int_{0}^{\infty} \mathscr{S}^{h}(B \cap\{f=t\}) d t \leq 2 \int_{B} \operatorname{lip}(f) d \mathfrak{m} .
$$

Proof. Let $B \subset X$ be a bounded Borel set. First we recall that if $\mathfrak{m}$ is a doubling measure then these two properties hold, which are both consequences of the Vitali covering theorem w.r.t. doubling measures:

(i) for all $\delta>0, \mathfrak{m}(B)=\inf \left\{\sum_{i} \mathfrak{m}\left(B_{i}\right): B_{i}=\bar{B}_{r_{i}}\left(x_{i}\right), r_{i} \in(0, \delta), B \subset \cup B_{i},\right\}$;

(ii) for every collection of closed balls $\mathcal{F}$ that is a fine cover of $B$ we have a disjoint collection $\mathcal{F}^{\prime} \subset \mathcal{F}$ that covers $\mathfrak{m}$-almost all of $B$.

For every $\delta>0$ and every $\varepsilon>0$ we have, by property (i), the existence of closed balls $B_{i}$ such that $B \subset \bigcup B_{i}$, radius $r_{i}<\delta / 2$ and $\mathfrak{m}(B) \leq \varepsilon+\sum_{i} \mathfrak{m}\left(B_{i}\right)$. Let us denote $t_{i}^{-}=\inf _{B_{i}} f$ and $t_{i}^{+}=\sup _{B_{i}} f$ : then $B_{i} \cap\{f=t\} \neq \emptyset$ implies that $t \in\left[t_{i}^{-}, t_{i}^{+}\right]$and in particular we have

$$
\mathscr{S}_{\delta}^{h}(B \cap\{f=t\}) \leq \sum_{i \text { s.t. } t \in\left[t_{i}^{-}, t_{i}^{+}\right]} \frac{\mathfrak{m}\left(B_{i}\right)}{2 r_{i}} .
$$

Integrating this inequality from 0 to $\infty$ we obtain

$$
\int_{0}^{\infty} \mathscr{S}_{\delta}^{h}(B \cap\{f=t\}) d t \leq \sum_{i} \frac{\mathfrak{m}\left(B_{i}\right)}{2 r_{i}}\left(t_{i}^{+}-t_{i}^{-}\right) .
$$

It is clear that $\left(t_{i}^{+}-t_{i}^{-}\right) \leq 2 r_{i} \operatorname{Lip}(f)$, and thus the countable subadditivity of $\mathscr{S}_{\delta}^{h}$ gives

$$
\int_{0}^{\infty} \mathscr{S}_{\delta}^{h}(B \cap\{f=t\}) d t \leq \operatorname{Lip}(f) \sum_{i} \mathfrak{m}\left(B_{i}\right) \leq \operatorname{Lip}(f)(\mathfrak{m}(B)+\varepsilon) .
$$

Letting $\varepsilon,, \delta \rightarrow 0$ we will get (5.1). This proves also that $\mathscr{S}^{h}(B \cap\{f=t\})=0$ for $\mathscr{L}^{1}$-a.e. $t \in(0, \infty)$ whenever $\mathfrak{m}(B)=0$; in this case, a fortiori, for every $\delta>0$ one has $\mathscr{S}_{\delta}^{h}(B \cap\{f=t\})=0$ for $\mathscr{L}^{1}$-a.e. $t \in(0, \infty)$.

Now we use property (ii): for every $\delta$ we find a a disjoint family of closed balls $\left\{B_{i}\right\}_{i \in \mathbb{N}}$ with radii less than $\delta / 2$ such that $\mathfrak{m}(B \backslash \tilde{B})=0$, where $\tilde{B}=\bigcup_{i} B_{i}$. It follows that

$$
\mathscr{S}_{\delta}^{h}(B \cap\{f=t\}) \leq \mathscr{S}_{\delta}^{h}(\tilde{B} \cap\{f=t\})+\mathscr{S}_{\delta}^{h}((B \backslash \tilde{B}) \cap\{f=t\})=\mathscr{S}_{\delta}^{h}(\tilde{B} \cap\{f=t\})
$$

for $\mathscr{L}^{1}$-a.e. $t \in(0, \infty)$ and thus $\int_{0}^{\infty} \mathscr{S}_{\delta}^{h}(B \cap\{f=t\}) d t=\int_{0}^{\infty} \mathscr{S}_{\delta}^{h}(\tilde{B} \cap\{f=t\}) d t$. Now we can apply (5.4) for $\tilde{B}$ and its covering to obtain the same inequality for $B$. Now we notice now that for all $x \in B_{i}$ one has

$$
\begin{aligned}
\frac{t_{i}^{+}-t_{i}^{-}}{2 r_{i}} & =\sup _{y, y^{\prime} \in B_{i}} \frac{f(y)-f\left(y^{\prime}\right)}{2 r_{i}} \leq \sup _{y, y^{\prime} \in B_{i}} \frac{|f(y)-f(x)|+\left|f\left(y^{\prime}\right)-f(x)\right|}{2 r_{i}} \\
& \leq 2 \sup _{0<\mathrm{d}(x, y) \leq \delta} \frac{|f(y)-f(x)|}{\mathrm{d}(y, x)}=: 2 \operatorname{sl}(x, \delta),
\end{aligned}
$$

where $\operatorname{sl}(\cdot, \delta)$ is the local slope on scale $\delta$, satisfying $\operatorname{sl}(x, \delta) \downarrow \operatorname{lip}(f)(x)$ as $\delta \downarrow 0$. In particular, using that $B_{i}$ are disjoint and cover $\mathfrak{m}$-almost all of $B$, we have deduce from (5.4) and the last inequality that

$$
\int_{0}^{\infty} \mathscr{S}_{\delta}^{h}(B \cap\{f=t\}) d t \leq 2 \int_{B} \operatorname{sl}(x, \delta) d \mathfrak{m}
$$


Now, we conclude letting $\delta \downarrow 0$ and using dominated convergence, since $\operatorname{sl}(\cdot, \delta) \leq \operatorname{Lip}(f)$. We can obtain the analogous result with $\operatorname{Lip}_{a}$ in the right hand side using that, for $x \in B_{i}$,

$$
\frac{t_{i}^{+}-t_{i}^{-}}{2 r_{i}} \leq \operatorname{Lip}\left(f, B_{i}\right) \leq \operatorname{Lip}\left(f, B_{2 \delta}(x)\right) \rightarrow \operatorname{Lip}_{a}(f, x) .
$$

Corollary 5.2 Let $(X, \mathrm{~d}, \mathfrak{m})$ be a doubling metric measure space, with $(X, \mathrm{~d})$ complete, such that (4.2) holds. Then for every Lipschitz function $f: X \rightarrow[0, \infty)$ such that $\mathfrak{m}(\{f>0\})<\infty$ the set $E_{t}=\{f \geq t\}$ is of finite perimeter and $P\left(E_{t}, \cdot\right) \geq \frac{1}{2} \mathscr{S}^{h}\left\llcorner\partial^{*} E_{t}\right.$ for $\mathscr{L}^{1}$-a.e. $t \in(0, \infty)$.

It would be nice to improve (5.1), replacing $\operatorname{Lip}(f) \mathfrak{m}(B)$ with $\int_{B} \operatorname{lip}(f) d \mathfrak{m}$ in the right hand side, (compare with (5.3)), but this seems difficult to obtain without extra assumptions. We notice also that anyhow this inequality is not optimal since, in the Euclidean case $X=\mathbb{R}^{d}$, we have $\mathscr{S}^{h}=\frac{\omega_{d}}{2 \omega_{d-1}} \mathscr{H}^{d-1}$ on rectifiable sets.

Finally, a natural question is the identification of regularity assumptions ensuring equality between Minkowski content and perimeter, somehow bypassing the question of connecting these notions to $\mathscr{S}^{h}\left\llcorner\partial^{*} E\right.$.

\section{References}

[AFP] L. Ambrosio, N. Fusco, D. Pallara, Functions of bounded variation and free discontinuity problems. Oxford University Press, 2000.

[Am1] L. Ambrosio, Some fine properties of sets of finite perimeter in Ahlfors regular metric measure spaces. Adv. Math., 159 (2001), 51-67.

[AM2] L. Ambrosio, Fine properties of sets of finite perimeter in doubling metric measure spaces. Set-Valued Anal., 10 (2002), 111-128.

[AmCaVi] L. Ambrosio, F. Capasso, E. Villa, Approximation of geometric densities of random closed sets, Bernoulli, 15 (2009), 1222-1242.

[AGS11a] L. Ambrosio, N. Gigli and G. Savaré, Calculus and heat flow in metric measure spaces and applications to spaces with Ricci bounds from below. Inventiones Mathematicae, 195 (2014), 289-391.

[AGS11b] L. Ambrosio, N. Gigli and G. Savaré, Metric measure spaces with Riemannian Ricci curvature bounded from below. Duke Math. J., 163 (2014), 1405-1490.

[AmDi] L. Ambrosio And S. Di Marino, Equivalent definitions of BV space and of total variation on metric measure spaces. Journal of Functional Analysis, 266 (2014), 41504188.

[AmPiSp] L. Ambrosio, A. Pinamonti, G. Speight, Tensorization of Cheeger energies, the space $H^{1,1}$ and the area formula. Advances in Mathematics, 281 (2015), 1145-1177.

[BuZa] Yu.D. Burago, V.A. Zalgaller, Geometric inequalities. Grundlehren der Mathematischen Wissenschaften [Fundamental Principles of Mathematical Sciences], 285. Springer Series in Soviet Mathematics. Springer-Verlag, 1988. 
[CaSb] L. Carbone, C. Sbordone, Some properties of $\Gamma$-limits of integral functionals. Ann. Mat. Pura Appl., 122 (1979), 1-60.

[CM15a] F. Cavalletti, A. Mondino, Sharp and rigid isoperimetric inequalities in metricmeasure spaces with lower Ricci curvature bounds, arXix:1502.06465.

[CM15b] F. Cavalletti, A. Mondino, Sharp geometric and functional inequalities in metric measure spaces with lower Ricci curvature bounds, arXix:1505.02061.

[CM15c] F. Cavalletti, A. Mondino, Isoperimetric inequalities for finite perimeter sets in metric-measure spaces with lower Ricci curvature bounds. In progress.

[ClL] A. Chambolle, S. Lisini, L. Lussardi, A remark on the anisotropic outer Minkowski content. Adv. in Calculus of Variations, 7 (2013), 241-266.

[Chee] J. CheEger, Differentiability of Lipschitz functions on metric measure spaces. Geom. Funct. Anal., 9 (1999), 428-517.

[Di] S. Di Marino, Recent advances on BV and Sobolev Spaces in metric measure spaces. PhD thesis, Scuola Normale Superiore, 2014 (available at cvgmt.sns.it).

[GRS] N. Gozlan, C. Roberto, P.-M. Samson, Hamilton Jacobi equations on metric spaces and transport entropy inequalities. Rev. Mat. Iberoam., 30 (2014), 133-163.

[GiHa] N. Gigli, B.-X. HAn, Independence on $p$ of weak upper gradients on RCD spaces. ArXiv 1407.7350.

[HKLl14] H. Hakkarainen, J. Kinnunen, P. Lahti, and P. Lehtelä, Relaxation and integral representation for functionals of linear growth on metric measure spaces. ArXiv 1401.5717 (2014).

[Led] M. Ledoux, The concentration of measure phenomenon. American Mathematical Society, 2001.

[Mi] M. Miranda JR, Functions of bounded variation on "good" metric spaces. J. Math. Pures Appl., 82 (2003), 975-1004.

[Ra] T. Rajala, Local Poincaré inequalities from stable curvature conditions on metric spaces. Calc. Var. Partial Differential Equations, 44 (2012), 477-494.

[Sa] G. SAVARÉ, Self-improvement of the Bakry-Émery condition and Wasserstein contraction of the heat flow in $\operatorname{RCD}(K, \infty)$ metric measure spaces. Discrete Contin. Dyn. Syst., 34 (2014), 1641-1661.

[HKST] J. Heinonen, P. Koskela, N. Shanmugalingham and J. Tyson, Sobolev spaces on metric measure spaces: an approach based on upper gradients. Cambridge University Press, New Mathematical Monographs 27 (2015). 